\title{
Emerging upconversion nanoparticles for industry and biomedical application
}

\author{
Evgeny Khaydukov ${ }^{1, *}$, Vasilina Rocheva ${ }^{1}$, Alexander Savelyev ${ }^{1}$, Kirill Khaydukov ${ }^{1}$, Ilya \\ Asharchuk ${ }^{1}$, Andrey Nechaev ${ }^{1,2}$, Dmitry Khochenkov ${ }^{1,3}$, Anastasia Sochilina ${ }^{1,4}$, Vladimir \\ Semchishen $^{1}$ and Alla Generalova ${ }^{1,4}$ \\ ${ }^{1}$ Federal Scientific Research Centre "Crystallography and Photonics" of the Russian Academy of \\ Sciences, Leninsky pr. 59, 119333 Moscow, Russia \\ ${ }^{2}$ Institute of Fine Chemical Technologies, Moscow Technological University, 86 Prospect \\ Vernadskogo, 119571 Moscow, Russia \\ ${ }^{3}$ N.N. Blokhin Russian Cancer Research Center, 115478, Moscow, Russia \\ ${ }^{4}$ Shemyakin-Ovchinnikov Institute of Bioorganic Chemistry of the Russian Academy of Sciences, \\ 16/10 Miklukho-Maklaya, 117997 Moscow, Russia
}

\begin{abstract}
In recent years, the overwhelming majority of the upconversion nanoparticles (UCNPs) prominent applications have originated from their unique luminescent properties. Due to original properties of inorganic UCNPs they attract the interest in numerous fields. We discussed a number of UCNP assisted techniques, such as biomedical imaging, therapy agents, anti-counterfeit labels and 3D printing, showing highly versatile and translatable UCNP photoluminescent nanotechnology for the applications in industry and biomedicine.
\end{abstract}

The main engineering advantage of UCNP platform, unlike conventional luminescent labels, is based on the nature of the anti-Stokes luminescence. UCNPs consist of an inorganic host matrix (usually $\mathrm{NaYF}_{4}, \mathrm{YF}_{3}, \mathrm{LiYF}_{4}$ ) co-doped with lanthanide ions act as the sensitizer (e.g., $\mathrm{Yb}^{3+}$ or $\mathrm{Nd}^{3+}$ ) and as the activator (e.g., $\mathrm{Er}^{3+} / \mathrm{Tm}^{3+}$ or $\mathrm{Ho}^{3+}$ ). The network of closely spaced sensitizer-ions resonantly absorbs NIR radiation and non-radiatively couples excitation energy to neighboring activator -ions. Activators provide the multiple photon absorption due to their ladder-like arranged levels and emit upconverted photons $[1]$.

\section{Near-infrared polymerization and security printing}

We demonstrated the effective and straightforward strategy for the NIR-activated polymerization of photocurable compositions containing UV-emitting upconversion nanomaterials. For this aim, the rationally-designed core/shell upconversion nanoparticles $\mathrm{NaYF}_{4}: \mathrm{Yb}^{3+}, \mathrm{Tm}^{3+} / \mathrm{NaYF}_{4}$, with the distinct ultraviolet-emitting lines have been used. Their ability to activate commercially available photoinitiators in the process of radical polymerization of light-sensitive resins at moderate NIR light intensities and irradiation

* Corresponding author: khaydukov@ mail.ru 
doses opens unique possibilities for the implementation of novel NIR triggered photopolymerization technique. This technique allows fabrication of $3 \mathrm{D}$ polymeric structures inside the volume of photocurable compositions.

We developed invisible biocompatible inks visualized under infrared irradiation for security printing applications. To produce stable water inks the surface of UCNPs was modified by amphiphilic polymer. Undetectable under daylight security printing was performed by ink-jet printing on paper. Upconversion of the printed patterns under near infrared laser irradiation was observed and imaged. Higher levels of security were achieved based on optical multiplexing capable combinations of $\mathrm{Ln}^{3+}$ in nanoparticles. The developed technology is promising for fast, easy and low cost inkjet printing for anticounterfeit and security applications.
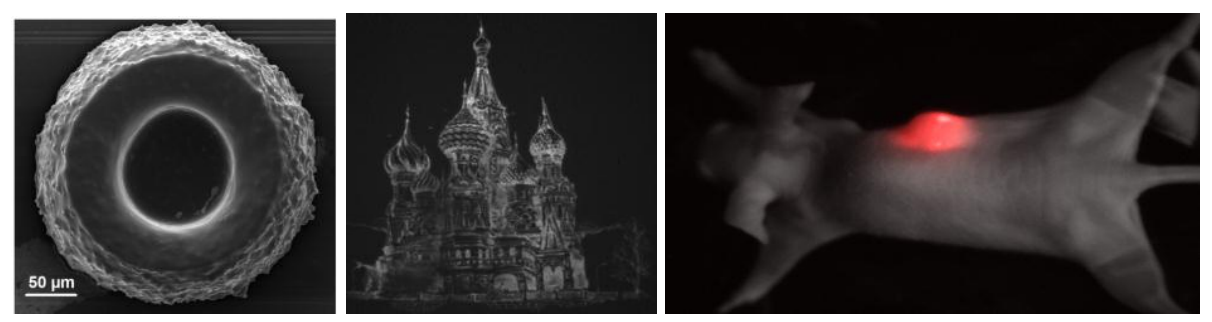

Fig. 1. Image of 3D polymer microstructure obtained by NIR-light-activated photopolymerization (left). The graphic image printed with upconversion inks (middle). UCNP biovisualization - the tumor exhibited high luminescent contrast (red color) demonstrating superiority of the UCNP-assisted imaging (right).

\section{Bioimaging and Photodynamic therapy}

Photodynamic therapy (PDT) is a clinical tumor treatment that utilizes cytotoxic reactive oxygen species (ROS), generated by photosensitizer, which is excited by external light source. However, conventional PDT is limited by the penetration depth of light required for photosensitizer activation. We demonstrate a solution to this problem by the rational photoluminescent nanotechnology design. Specifically, upconversion nanoparticles (UCNPs) offer a lucrative possibility to kindle UV or visible light at the sub-centimetre depth in tissue, owing to their property to convert infrared radiation at the wavelength in the biological tissue transparency window $975 \mathrm{~nm}$. We designed upconversion nanoparticles (UCNP) of the composition $\mathrm{NaYF}_{4}: \mathrm{Yb}^{3+}: \mathrm{Tm}^{3+}$ that yielded $\mathrm{UV} /$ blue emission bands critical for the photosensitising of vitamin $\mathrm{B}_{2}$. We achieved a high Förster resonance energy transfer (FRET) efficiency from UCNPs to vitamin $\mathrm{B}_{2}$, which facilitates a large production of cytotoxic singlet oxygen and thus an enhanced PDT efficacy. Design UCNPs was used as PL probes for tumor labelling. The comparison of the image acquisition in the Stokes and anti-Stokes channels shows much greater contrast in the anti-Stokes channel. This clearly demonstrates the advantage of UCNP-assisted imaging.

This scientific work has been partly supported by Grants RSF No. 16-13-10528 (in the part of UCNP assisted NIR-activated polymerization), RFBR No. 17-00-00122 (K) (in the part of UCNP assisted bioimaging and therapy).

\section{References}

1. A. N. Generalova, B. N. Chichkov, E. V. Khaydukov, Adv. Colloid Interface Sci. 245, 1 (2017). 